\title{
Fuzzy based Enhanced Clustering Protocol using Mitigation for Multi-Hop in WSN
}

\author{
Preeti Saroj \\ M.Tech Student \\ Department of Computer science and Engineering \\ Uttarakhand Technical University Dehradun
}

\begin{abstract}
In Wireless Sensor Networks (WSN) nodes communicate with each other and their Base Station(BS). Since, the networks are battery operated and are deployed in challenging areas utilizing the resources effectively is a must. Clustering solves the issue of resource allocation to a great deal with proper selection of the cluster head $(\mathrm{CH})$. The proposed work uses Mamdani Fuzzy interface. The fuzzy rules are defined on the basis of Centrality of the node, bandwidth, energy and probability. Once the $\mathrm{CH}$ is selected and task assigned to the node a mitigation process is proposed to reallocate the nodes with the resources and update the network. The updated resources manages the energy and bandwidth more efficiently without compromising the time. The results observed confirms that the proposed algorithm performs better than SEP, TSEP, ETSEEP, and base algorithms in terms of energy, time and throughput.
\end{abstract}

\section{General Terms}

The proposed work is improvement in energy efficiency of the wireless sensor network and using mitigation for resource reallocation and thus improving the network lifetime, efficient energy using fuzzy logic and mitigation using for enhance network lifetime.

\section{Keywords}

Energy efficiency, fuzzy type, mitigation, WSN

\section{INTRODUCTION}

Wireless sensor network is self-organization network. It have various type of tiny sensor nodes which are communication between themselves with the help of radio signals and locate in capacity to sense, processing and monitor to physical world. Sensor nodes are also called 'motes'. WSN is a communication link between real physical and virtual world. It has a broad range of a probable application to the security, industries, science, civil infrastructure and transportation. A sensor node might vary in size from that of a shoebox down to the size of a grain of dust, although functioning "motes" of genuine microscopic dimensions. The cast of sensor node is similarly variable ranging from hundred of dollar to a few pennies depending on the complexity of the individual sensor nodes. It has size and cost constraints on resource such as energy memory, computation speed and communication bandwidth. Energy consumption in WSN is very important issues enhancing network lifetime. In WSN there are no resource for recharging a battery power and when manufacture of devices, large sized battery are avoided because sensor node are very tiny and nodes have limited power capacity. Sensor nodes are remotely deployed in battery operated in WSN, So that when we formation of the cluster one higher energy node and some other type of major quality have nodes elects as $\mathrm{CH}$.

\author{
Sanjay Kumar, PhD \\ Assistant Professor \\ Department of Computer Science and Engineering \\ Uttarakhand Technical University Dehradun
}

\section{RELATED WORK}

In this chapter we proposed work. In this section is analysis our proposed scheme, network model, algorithm, and flowchart. WSNs are extensively used in control disaster recovery, traffic control, monitor environmental things and home security. WSNs have a sensor node, which have ability to collect information and forward it to BS. The major term in WSN is clustering routing algorithm, which is very effectively work to deal with network energy utilization and also topology regarding problems. The first energy efficiency protocol is LEACH (Low Energy Adaptive Clustering Hierarchy) and LEACH is a hierarchal clustering algorithm which is used random election of $\mathrm{CH}$ in network [1]. Energy is very important criteria in cluster formation, cluster head election method during the energy of the whole network is weighed down to every sensor node, and network lifetime is also enhanced. Kim $\mathbf{J}$ M et al. is proposed a CHEF (cluster head election mechanism based on fuzzy logic) in WSNs [6], this technique mainly elects the cluster head considering by residual energy of each node and the distance among the node to the base station, but one drawback of this is neglects the pressure of the number of neighboring node in the entire network. EAMMH is a dispersed clustering multi-hop routing protocol in WSNs, it is mainly concern elects of $\mathrm{CH}$ and it is considered three parameters based on the residual energy, multi-hop communication whole the information gathering. So that multi-hop data transference is perform by inter-cluster to expand the continued existence time in the network. Multihop transmission is generally used for the inter-cluster communication and intra-cluster is fault produce to the hot zone problem. Alaybeyoglu A et al proposed a DFLC and DFLC algorithm is uses fuzzy logic interference set to choose $\mathrm{CH}$. Multi-hop transmission information is used in communication and data formation and data gathering in the intra-cluster. The algorithm is used three parameters for decide to node elects as $\mathrm{CH}$ the residual energy of nodes, the distance to base station(BS) each node, and the node density as the reason for $\mathrm{CH}$ election. However, the DFLC algorithm is disregard the hot zone difficulty in multi-hop communication. However, in every rounds of real-time communication, the nodes have to be communicating with the BS to obtain the distance to the BS, which enlarge the supplementary energy utilization of the nodes. Zhang Siquing et al proposed FLCMN algorithm, which is basically, used fuzzy set for election of $\mathrm{CH}$ with using residual energy, number of neighbour nodes and average residual energy of neighbour nodes. FLCMN is considered hotspot problem and energy consumption. But in this approach considered inter cluster by using multi-hop communication that is contains more load in the network and also contain high-energy consumption.

However, our proposed approach is consistent for energy efficiency in the network and also prolong network lifetime. 
Our proposed algorithm is used fuzzy logic for a reliable $\mathrm{CH}$ election probability using with residual energy of each node, density, centrality of each node and distance between every node and base station in the network and mitigation is used for elaborate the energy consumption in the network and when the network fail it recover it reallocation of all nodes and their resource too.

\section{NETWORK MODEL}

- We used hierarchical clustering to distribute the entire network into many clusters.

- $\quad$ There are selecting one node with higher energy as a $\mathrm{CH}$ in each cluster.

- $\quad$ Each sensor node directly connected to $\mathrm{CH}$ and also each $\mathrm{CH}$ direct connected to $\mathrm{BS}$ (sink node).

- Sensor nodes are sending all data to nearby $\mathrm{CH}$ in a cluster and every $\mathrm{CH}$ send all aggregate data to BS.

- Every $\mathrm{CH}$ maintains the information data with each node in entire cluster and network too.

- Mitigation is using in our network that are generally used for reallocation of nodes with the resource.

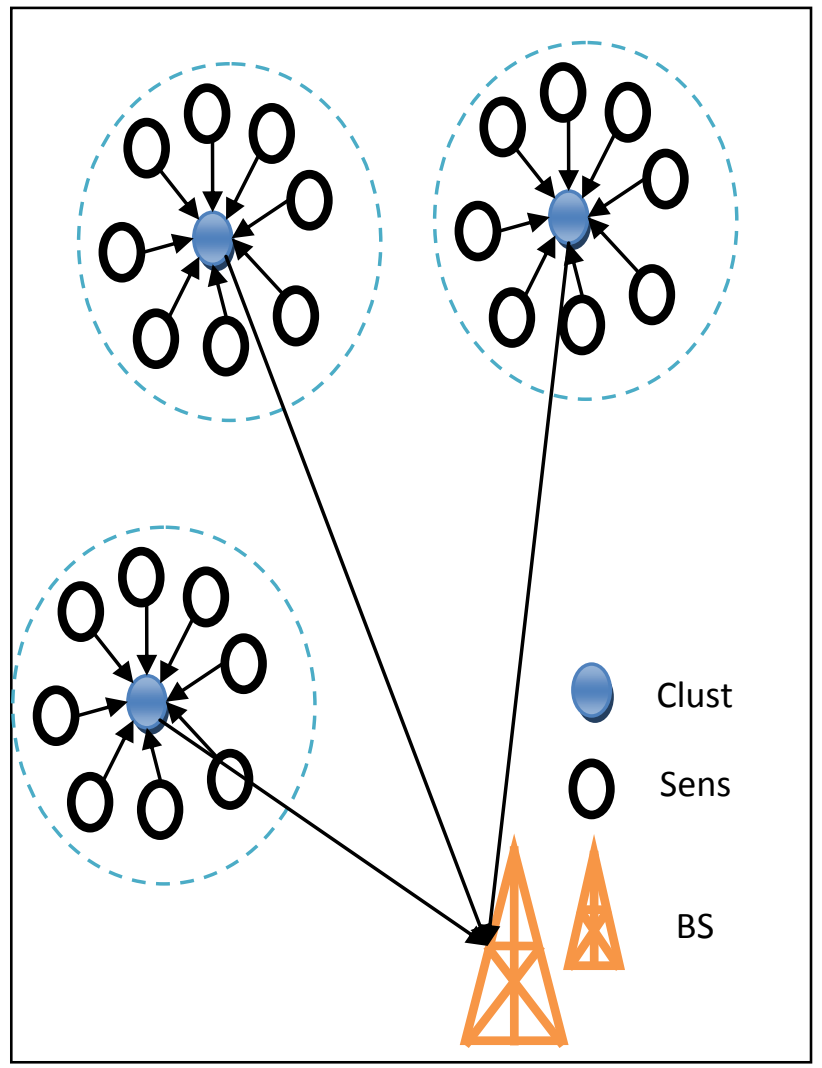

\section{PROPOSED MODEL}

Proposed algorithm F-ECPM is based on reactive routing protocol. $\mathrm{CH}$ election by fuzzy logic using if then else operations are making less energy consume and lifetime increase in WSN. There is $\mathrm{CH}$ dynamically chosen in each round because when one cluster head die another one take place of a current cluster head. We are using three phase in our proposed algorithm that are first phase custer formation, second phase cluster head selection by fuzzy interface system and last phase is mitigation.

\subsection{Phase First (Cluster Formation)}

The static cluster formation is performed only once at the beginning of network operation alike as LEACH. During the cluster formation, entire network was distributing into various clusters. Each cluster consist one cluster head node and many sensor nodes. Each sensor node directly connected to nearby $\mathrm{CH}$ in the cluster. Cluster head is directly connects to base station (sink node).

\subsection{Phase second (cluster head selection)}

In this phase cluster head $(\mathrm{CH})$ election starts. Initially, each sensor nodes have the same energy. Thus, loop start in first round any sensor node from each cluster have higher energy in comparison of other sensor node can be selected as cluster head for that cluster. This process is done by fuzzy interference system, which is give a reliable probability of each sensor nodes to be elects as $\mathrm{CH}$. When the fuzzy interference is give the output probability then analysis that $\mathrm{CH}_{\mathrm{ij}}$ is less than balance energy and bandwidth also. If satisfy this condition elects as $\mathrm{CH}$ in current round and send the acceptance massage to $\mathrm{BS}$. $\mathrm{CH}_{\mathrm{ij}}$ is send acknowledgement massage to all nodes in entire cluster to join it.

\subsection{Mitigation phase}

In the mitigation phase is use of energy balancing in entire network. Mitigation is reallocation of node by reserve energy and updates the entire network.

\subsection{Algorithm}

START

1. Loop till true.

2. msgreceive become form sensor.

3. Get location of the sensor.

4. Get number of neighbouring nodes.

5. Get the central location of the node $\leftarrow$ centrality

6. Calculate density of the area selected

7. Get probability of node to be selected as $\mathrm{CH} \leftarrow$ probable $\mathrm{CH}$

8. Get energy of the Probable $\mathrm{CH}$

9. Get bandwidth of the Probable $\mathrm{CH}$ probability)

11. $\mathrm{RE} \leftarrow$ Calculate required energy

12. $\mathrm{BW} \leftarrow$ Calculate required bandwidth

13. $\mathrm{CH}_{\mathrm{ij}} \leftarrow$ Get node number of the output returned by Fuzzy

14. If $\mathrm{RE}$ is less than Balance_energy of $\mathrm{CH}_{\mathrm{ij}}$ and $\mathrm{BW}$ is less than Balance_Bandwidth

15. Select it as $\mathrm{CH}$ and send acceptance to Base Station with information of the node

16. Update resources of the node

17. Else

18. Repeat steps 2 through 14

19. End if

20. Loop through number of sensors //mitigation 
21. $\mathrm{re}_{\mathrm{i}} \leftarrow$ record remaining energy of the node

22. $\mathrm{bw}_{\mathrm{i}} \leftarrow$ record remaining bandwidth

23. If sensor not in use

24. $\quad$ Sensor $_{\mathrm{ij}} \leftarrow$ Sensor $_{\text {nearest }} \quad / /$ Transfer request of nearest neighbour

25. Update resources of the Sensor nearest

26. Endif

27. End loop

28. End loop

29. Calculate remaining resources

\section{RESULTS}

We have introduced faults in rounds multiple of $11^{\text {th }}, 13^{\text {th }}$ and $17^{\text {th }}$. The faulty rounds are prime numbers which are selected to provide faults at a steady manner in order to study them for long course of network simulation .In round $11^{*} 13=143$ th both $\mathrm{CH} 1$ and $\mathrm{CH} 2$ goes down and recovered. In $11 * 17=187^{\text {th }}$ round both $\mathrm{CH} 1$ and $\mathrm{CH} 3$ goes down and cluster is recovered.

\subsection{Parameters}

$\mathrm{F}$ This section is assessing represent simulation performance of the F-ECPM algorithm and comparing F-ECPM with SEP, TSEP, ETSSEP, and FLCAM. MATLAB is used to builds simulation work and carried out all types of fuzzy MFs are available in it. These Parameters are showing in below Table 5.1. This table is representing what kind of parameters and their values we use in our proposed approach F-ECPM. These parameters are evaluate our proposed approach setup phase and these parameters are generalized energy given each node, bandwidth of nodes, data packet size and base station location. We have considered 100 sensor nodes are distributing in $200 \times 200 \mathrm{~m}$ area. F-ECPM approach $\mathrm{CH}$ selection is based on fuzzy logic and using four fuzzy descriptors such as residual energy, centrality, density and bandwidth.

following are the parameters used in the simulation

Table 5.1 Simulation parameters

\begin{tabular}{|c|c|}
\hline PARAMETERS & VALUES \\
\hline Network field size & $\begin{array}{cc}\mathrm{x}=200 & \text { meters } \\
\mathrm{y}=200 \text { meters } & \end{array}$ \\
\hline $\begin{array}{l}\text { Number of sensor nodes } \\
\text { used }\end{array}$ & 100 \\
\hline $\begin{array}{c}\text { Base station(Sink node) } \\
\text { location }\end{array}$ & $(100,50)$ \\
\hline Initial energy of nodes & $0.3 \mathrm{~J}$ \\
\hline $\mathrm{E}_{\mathrm{DA}}$ & $5 * 10-9 \mathrm{~J}$ \\
\hline Efs & $10-11 \mathrm{~J}$ \\
\hline Elec & $5 * 10-8 \mathrm{~J}$ \\
\hline Emp & $1.3 * 10-13 \mathrm{~J}$ \\
\hline Bandwidth & $1 \mathrm{Mbps}$ \\
\hline Data packet size & 400bits \\
\hline Number of Rounds & 600 \\
\hline
\end{tabular}

- Residual Energy: Residual energy is very impotent factor that is used in electing cluster head and cluster head node have more energy in comparison to other member node. Cluster head collects all data to member nodes and send the aggregate data to base station so it required more energy. Residual energy is calculating by equation (1).

$$
R E=\sum_{i=0}^{100}(E-\mu / n)
$$

- Density : Density is mainly degree of compactness of substance that is divide factor between number of node neighbours and number of node.

Density $_{\mathrm{i}}=\frac{(\text { number } \text { of node neighbour } \mathrm{s})}{(\text { number of node })}$

- Centrality: Centrality is contains all nodes centrality in sensor network.

centrality $_{\mathrm{i}}[1]=\sqrt{\sum_{\text {node }_{j} \text {-node }} \text { dist }_{i}^{2}(i, j) /\left(\mid \text { node }^{\prime} \text { neighbours }(i) \mid\right)}-$ node

- Bandwidth: Bandwidth is amount of data that can be transmitted in one point to another over in sensor network in fixed amount of time.

$R B W=\sum_{i=0}^{100}(B W-\lambda / n)$ eq

Where $\gamma=$ required bandwidth of the signal.

The residual energy of the nodes

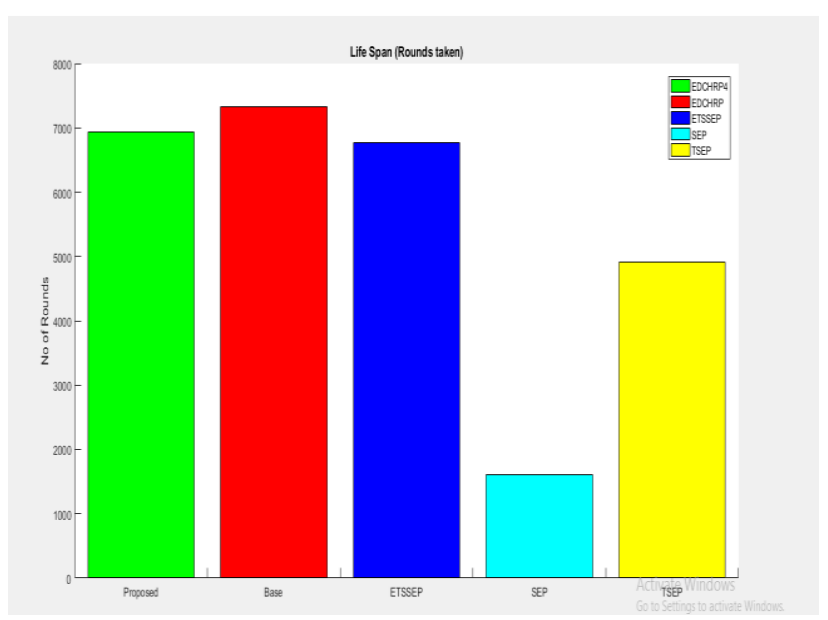

Figure 5.1 network lifespan result all algorithm 


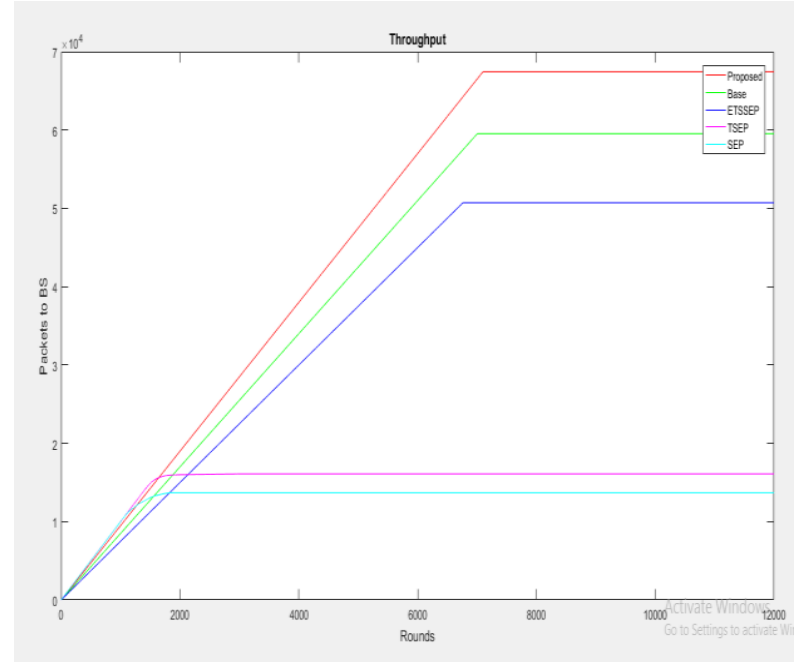

Figure 5.2 Throughput result all algorithm

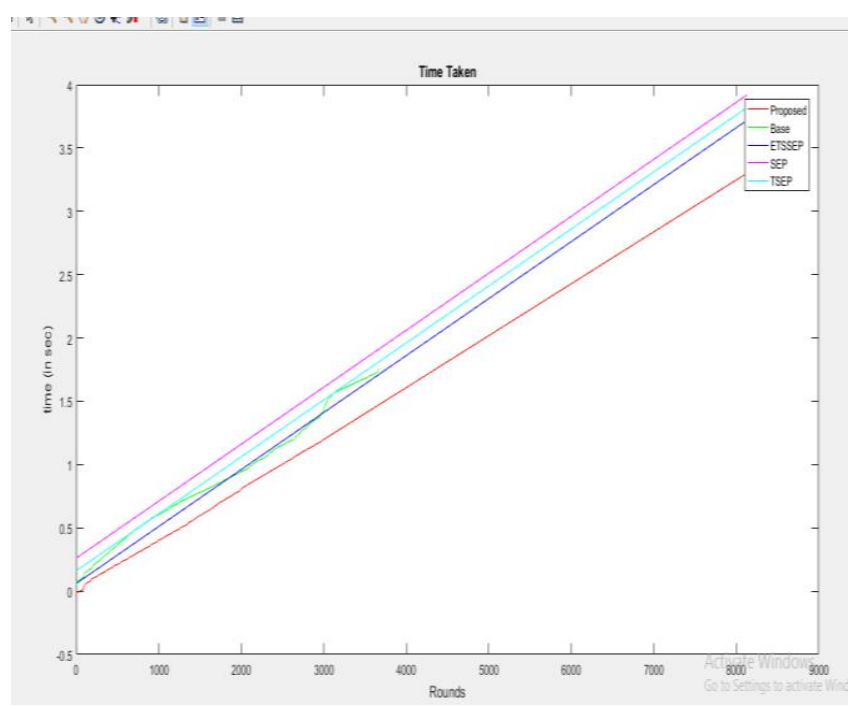

Figure 5.3 Time span result all algorithm.

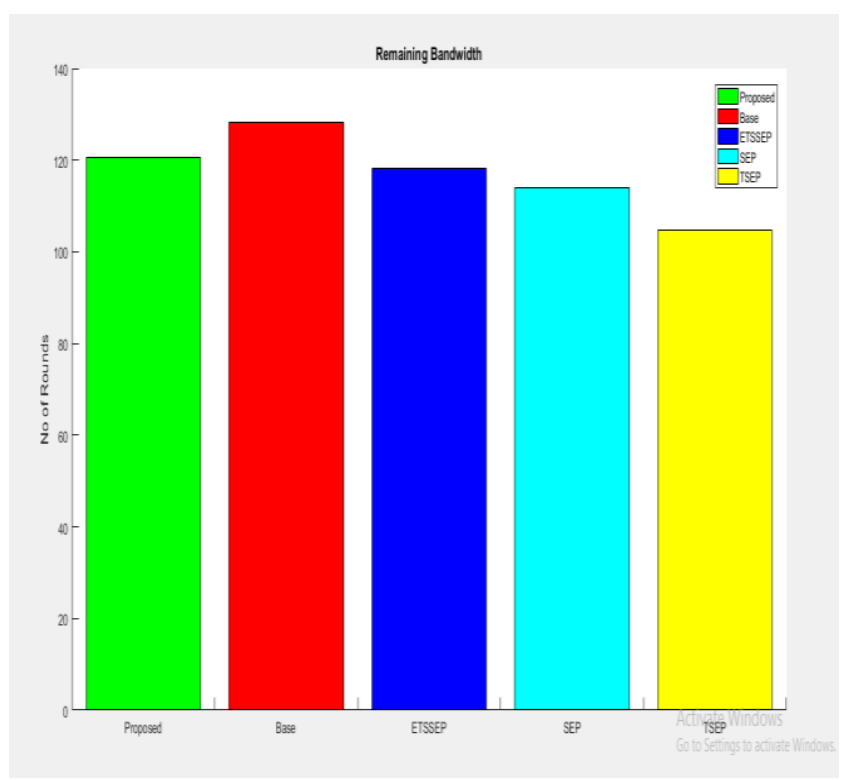

Figure 5.4 remaining bandwidth result all algorithm

\section{CONCLUSION \& FUTURE WORK}

In this proposition, we displayed a modified version of clustering approach using fuzzy logic and mitigation. F-ECPM algorithm is also compared with all other algorithm SEP, TSEP, ETSSEP and FLCMN algorithms. F-ECPM approach outperformed the SEP, TSEP, ETSSEP and FLCMN algorithms in terms of bandwidth consumption, time and energy consumption. The bandwidth is improved by $15 \%$, energy by $18 \%$ and time by $6 \%$. MATLAB tool is used for implementation purpose. In future the model can be tested over nerofuzzy and predictive modelling may also be tried using Machine Learning (ML) and also hotspot problem. The work presented in the thesis has been inspired by the energy constraint.

\section{REFERENCES}

[1] W. R. Heinzelman, A. Chandrakasan, and $\mathrm{H}$ Balakrishnan, "Energyefficient communication protocol for wireless microsensor networks," in Proc. 33rd Hawaii Int. Conf. Syst. Sci. (HICSS), Washington, DC, USA, Jan. 2000, pp. 1-10.

[2] W. B. Heinzelman, A. P. Chandrakasan, and H. Balakrishnan, "An application-specific protocol architecture for wireless microsensor networks," IEEE Trans. Wireless Commun., vol. 1, no. 4, pp. 660-670, Oct. 2002.

[3] S. Lindsey and C. S. Raghabendra, "PEGASIS: Power efficient gathering in sensor information systems," in Proc. IEEE Aerosp. Conf., Mar. 2002, pp. 3-1125-31130.

[4] I. Gupta, D. Riordan, and S. Sampalli, "Cluster-head election using fuzzy logic for wireless sensor networks," in Proc. Commun. Netw. Services Res. Conf., May 2005, pp. 255260.

[5] J.-M. Kim, S.-H. Park, Y.-J. Han, and T. Chung, "CHEF: Cluster head election mechanism using fuzzy logic in wireless sensor networks," in Proc. ICACT, Feb. 2008, pp. 654-659.

[6] A. Alkesh, A. K. Singh, and N. Purohit, "A moving base station strategy using fuzzy logic for lifetime enhancement in wireless sensor network," in Proc. Int. Conf. Commun. Syst. Netw. Technol., Jun. 2011, pp. 198-202.

[7] H. Taheri, P. Neamatollahi, O. M. Younis, S. Naghibzadeh, and M. H. Yaghmaee, "An energy-aware distributed clustering protocol in wireless sensor networks using fuzzy logic," Ad Hoc Netw., vol. 10, no. 7, pp. 1469-1481, 2012.

[8] T. Sharma and B. Kumar, "F-MCHEL: Fuzzy based master cluster head election leach protocol in wireless sensor network," Int. J. Comput. Sci. Telecommun., vol. 3, no. 10, pp. 8-13, Oct. 2012.

[9] Izadi, D., Abawajy, J. and Ghanavati, S. (2013). A new energy efficient cluster-head and backup selection scheme in WSN. 2013 IEEE 14th International Conference on Information Reuse \& Integration (IRI).

[10] Saber AMRI, Med Lassaad KADDACHI, Abdelbasset TRAD (2014) Energy-Efficient Multi-hop Hierarchical Routing Protocol using Fuzzy Logic (EMHR-FL) for WSN. 978-1-4799-3351-8/14/\$31.00 @2014 IEEE.

[11] Hassan EL ALAMI, Abdellah NAJID (2016) EnergyEfficient Fuzzy Logic Cluster Head selection in Wireless 
Sensor Networks. 978-1-4673-7689-1/16/\$31.00 @2016 IEEE.

[12] Padmalaya Nayak, Bhavani Vathasavai (2017) Energy efficency clustering algorithm for multi-hop wireless sensor network using type-2 fuzzy logic. IEEE sensor journal VOL.17,NO.14,JULY 15,2017.

[13] Z. W. Siew, C. F. Liau, A. Kiring, M. S. Arifianto, and K. T. K. Teo, "Fuzzy logic based cluster head election for wireless sensor network," in Proc. 3rd CUTSE Int. Conf., Miri, Malaysia, Nov. 2011, pp. 301-306.

[14] V. Nehra, R. Pal, and A. K. Sharma, "Fuzzy-based leader selection for topology controlled PEGASIS protocol for lifetime enhancement in wireless sensor network," Int. J. Comput. Technol., vol. 4, no. 3, pp. 755-764, Mar./Apr. 2013.

[15] G. Ran, H. Zhang, and S. Gong, "Improving on LEACH protocol of wireless sensor networks using fuzzy logic," J. Inf. Comput. Sci., vol. 7, no. 3, pp. 767-775, 2010.

[16] H. Ando, L. Barolli, A. Durresi, F. Xhafa, and A. Koyama, "An intelligent fuzzy-based cluster head selection system for WSNs and its performance evaluation for D3N parameter," in Proc. Int. Conf.
Broadband, Wireless Comput., Commun. Appl., Nov. 2010, pp. 648-653.

[17] Animesh Shrivastava and Singh Rajawat," An Implementation of Hybrid Genetic Algorithm for Clustering based Data for Web Recommendation System,"International journal of computer science and engineering, Volume-2, Issue -4, April 2014.

[18] Z. Arabi, "HERF: A hybrid energy efficient routing using a fuzzy method in wireless sensor networks," in Proc. Int. Conf. Intell. Adv. Syst. (ICIAS), Jun. 2010, pp. $1-6$.

[19] Shekhar Kumar, Shashi Kant Verma, Awadhesh Kumar (2015). Enhanced Threshold Sensitive Stable Election Protocol for Heterogeneous Wireless Sensor Network. December 2015, Volume 85, Issue 4, pp 2643-2656.

[20] E. H. Mamdani and S. Assilian, "An experiment in linguistic synthesis with a fuzzy logic controller," Int. J. Man-Mach. Stud., vol. 7, no. 1, pp. 1-13, 1975.

[21] K. Akkaya and M. Younis, "A survey on routing protocols for wireless sensor networks," Ad Hoc Netw., vol. 3, no. 3, pp. 325-349, 2005. 